\title{
ON THE IMPROVEMENT OF ASCAT WIND DATA ASSIMILATION IN GLOBAL NWP
}

\author{
Wenming Lin ${ }^{1}$, Marcos Portabella ${ }^{1}$, Ad Stoffelen ${ }^{2}$, Giovanna De Chiara ${ }^{3}$, Justino Martínez $^{1}$ \\ ${ }^{1}$ Institut de Ciències del Mar (ICM-CSIC), Barcelona, Spain. \\ ${ }^{2}$ Royal Netherlands Meteorological Institute (KNMI), De Bilt, The Netherlands \\ ${ }^{3}$ European Center for Medium range Weather Forecasting (ECMWF), Reading, UK
}

\begin{abstract}
The assimilation of Advanced Scatterometer (ASCAT) winds has proven to be beneficial for the European Center for Medium Range Weather Forecasting (ECMWF) system, particularly over the Tropics. In this study, several important aspects of the ASCAT data are addressed in order to further test and improve the impact of scatterometer wind data assimilation into ECMWF Integrated Forecasting System (IFS). First, an improved wind quality control (QC) is proposed and used to remove unrepresentative ASCAT winds. Second, a new ASCAT wind product, more representative of the ECMWF model resolved scales, is produced by averaging the relatively-high resolution ASCAT wind vector cells to lower resolution in an aggregation process. Two months of ASCAT low resolution data are then used to evaluate the impact of the refined QC and the aggregation technique on the IFS data assimilation.
\end{abstract}

Index Terms - Scatterometer, winds, data assimilation, quality control, representativeness error

\section{INTRODUCTION}

The retrieved wind field data from the Advanced Scatterometers onboard Metop, i.e., ASCAT-A and ASCAT-B, have been assimilated into the European Center for Medium Range Weather Forecasting (ECMWF) Integrated Forecasting System (IFS) since June 2007 and July 2013, respectively. In particular, the ASCAT wind product at $25 \mathrm{~km}$ grid resolution, with an estimated wind component error value of $1.5 \mathrm{~m} / \mathrm{s}$ and thinned every $100 \mathrm{~km}$ (which reduces the number of assimilated observations by a factor of 16, while retaining the essential information of the data), is assimilated [1]. Several attempts to improve the impact of ASCAT wind data on the IFS assimilation system have been recently carried out. For instance, it is proposed to assimilate at higher grid resolution or reduced thinning, in which case the ASCAT observation error should be inflated [2]. It is also proposed to use the situation-dependent observation (O) and background (B) error variances instead of the fixed $\mathrm{O} / \mathrm{B}$ errors, following the successful application in the ASCAT Two Dimensional Variational ambiguity removal (AR) scheme (2DVAR) [3][4]. However, as reported by Stoffelen et al. [5], several other important issues also need to be addressed in order to improve the impact of scatterometer wind data assimilation into global Numerical Weather Prediction (NWP) models.

The removal of unrepresentative winds using an improved wind Quality Control (QC) is in demand. A more aggressive QC may bring higher forecast impact, but not necessarily a closer analysis fit to ASCAT. Since the inversion residual or Maximum Likelihood Estimator (MLE) is a good proxy for local wind variability, it is strongly correlated with high observation minus background (O-B) departures. Usually, unrepresentative observations are handled by a first guess check, but this depends on the highly variable quality of the background in such highly variable cases, which correspond to squall lines, gust fronts, etc. A more independent and observation-based QC, based on the MLE and Singularity Exponent (SE) indices needs to be tested [3].

Another important aspect of scatterometer wind data assimilation is the spatial resolution. The development of a new ASCAT wind product more representative of the NWP model resolved scales should lead to improved data assimilation impact. In global scatterometer wind data assimilation, particularly in the Tropics, the spatial representativeness error (i.e., the true small-scale variance resolved by scatterometers but not by NWP models) is quite variable and spatially correlated. To avoid such spatial correlation, at the moment, scatterometer observations are thinned and assimilated with a relatively low weight, and as a consequence the small-scale information in scatterometer winds is not assimilated. A way to reduce the spatial representativeness error (and thus, its associated spatial correlation) is by averaging the relatively-high resolution ASCAT wind vector cells (WVCs) to lower resolution in an aggregation process. Such aggregated winds can be assimilated without thinning and lead to improved data assimilation impact.

This study mainly focuses on the above tasks. Section 2 reviews two essential quality indicators and their application in wind quality control and quality classification. In section 3 , an aggregation process is described and applied to generate new ASCAT wind products which are more representative of the ECMWF resolved (wind) scales than 


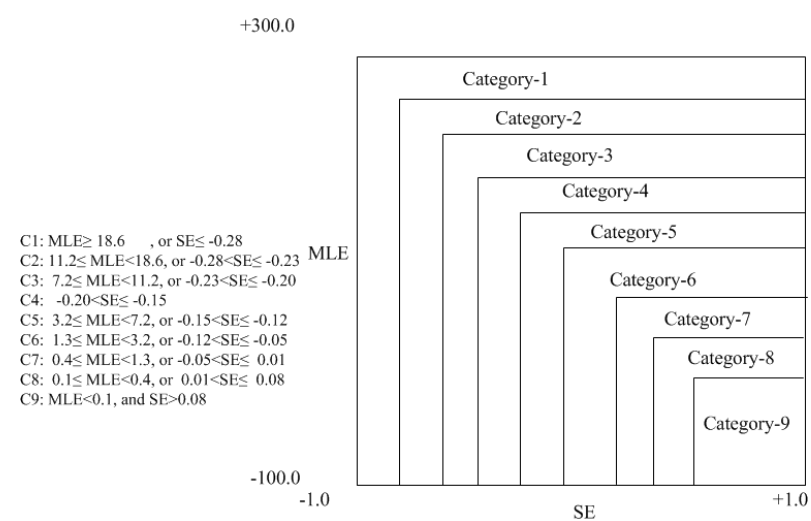

Fig. 1 Schematic representation of the defined categories used to develop situation-dependent O/B errors for the collocated ASCAT $25-\mathrm{km}$ data set. The numerical intervals of SE and MLE are presented in the $x$ - and $y$-axis respectively.

the nominal product. Then a triple collocation analysis is adopted to quantify the spatial representativeness errors and the standard deviation errors of wind zonal $(u)$ and meridional $(v)$ components. In section 4 , the experiments associated with IFS data assimilation are presented. Finally, the conclusions can be found in Section 5.

\section{WIND DATA QUALITY CONTROL AND CLASSIFICATION}

The wind QC method is developed by analyzing the characteristics of certain quality-sensitive parameters derived from the scatterometer data itself, and by optimizing the thresholds of these parameters in order to preserve (filter) as many representative (unrepresentative) winds as possible. A commonly used QC indicator is the inversion residual or maximum likelihood estimator (MLE). In general, the measurement sets are located close to the Geophysical Model Function (GMF) surface, leading to low MLE values and good quality wind retrievals. Occasionally, a large inconsistency between the triplets and the GMF is induced by other geophysical conditions than a WVC-mean wind, resulting in large MLE values.

An image processing technique, known as singularity analysis (SA), has proven to be more effective than MLE in terms of detecting the presence of rain [6]. The singularity exponents (SEs) derived from SA provide quantitative information about the sub- and/or inter-WVC variability, and are a complementary QC indicator to MLE. The combination of SE and MLE has been proposed to improve the ASCAT wind QC, as well as to to classify and quantify the buoy, ASCAT and NWP wind uncertainty as a function of wind variability, based on triple collocation (TC) analysis [3]. In particular, a new SA implementation scheme, which combines the gradient measurements of multiple parameters

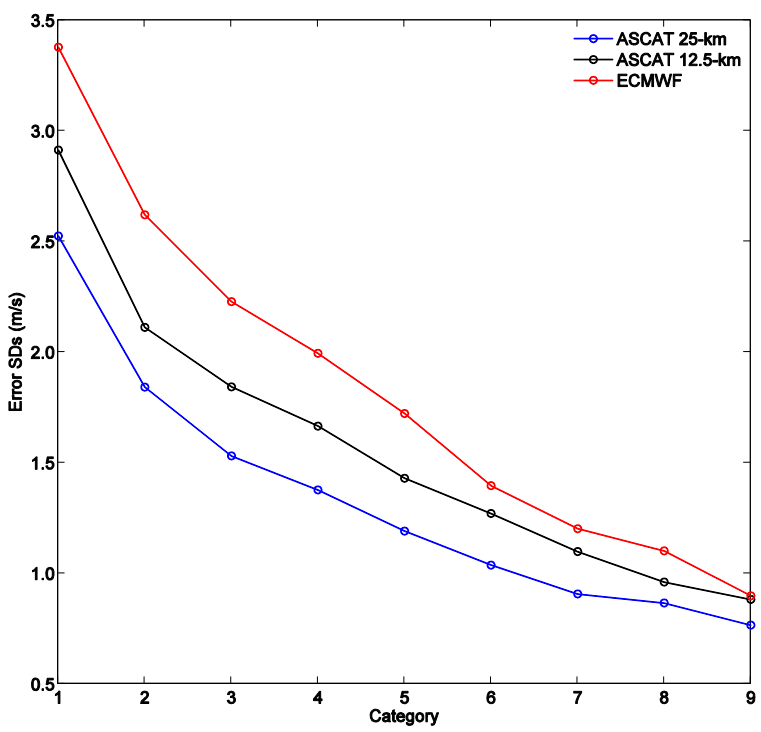

Fig. 2 The estimated error SDs of ASCAT 25-km (blue), ASCAT 12.5-km (black) and ECMWF (red) winds on the scale resolved by ECMWF for different wind categories.

in the analysis, is used to reveal the underlying geophysical phenomena.

In [3], MLE and SE intervals were used to classify the wind quality of the ASCAT $12.5-\mathrm{km}$ product in different wind quality categories. However, the ASCAT data set was only separated into 6 categories due the limited amount of collocations. In addition, the categorization was not optimized. The quality categorization is further refined in this section using the ASCAT-derived MLE and SE with an extended collocation data set. To separate the data set in a more meaningful way than [3], several criteria are used to determine the categorization. For instance, it is proposed to separate the collocated ASCAT 25-km data set into 9 categories (see the corresponding MLE/SE intervals in the left side of Fig. 1). A similar separation is applied to the collocated ASCAT 12.5-km data set, in which MLE/SE intervals are somewhat different than those shown in Fig. 1, but the category percentiles are similar to those of the $25-\mathrm{km}$ product. Fig. 2 shows the estimated error SDs (i.e., $\sqrt{\frac{S D_{u}^{2}+S D_{v}^{2}}{2}}$; on ECMWF scale) for ASCAT and ECMWF winds under different wind categories. Note that the ECMWF errors are independent of the ASCAT grid resolution.

\section{NEW ASCAT PRODUCTS}

The current thinning strategy (i.e., one wind observation used every $100 \mathrm{~km}$ ) does not assimilate the small-scale information in scatterometer winds, and does not reduce the inherent representativeness error of ASCAT observations. The latter is a particularly relevant issue that can potentially degrade the impact of scatterometer data assimilation into 

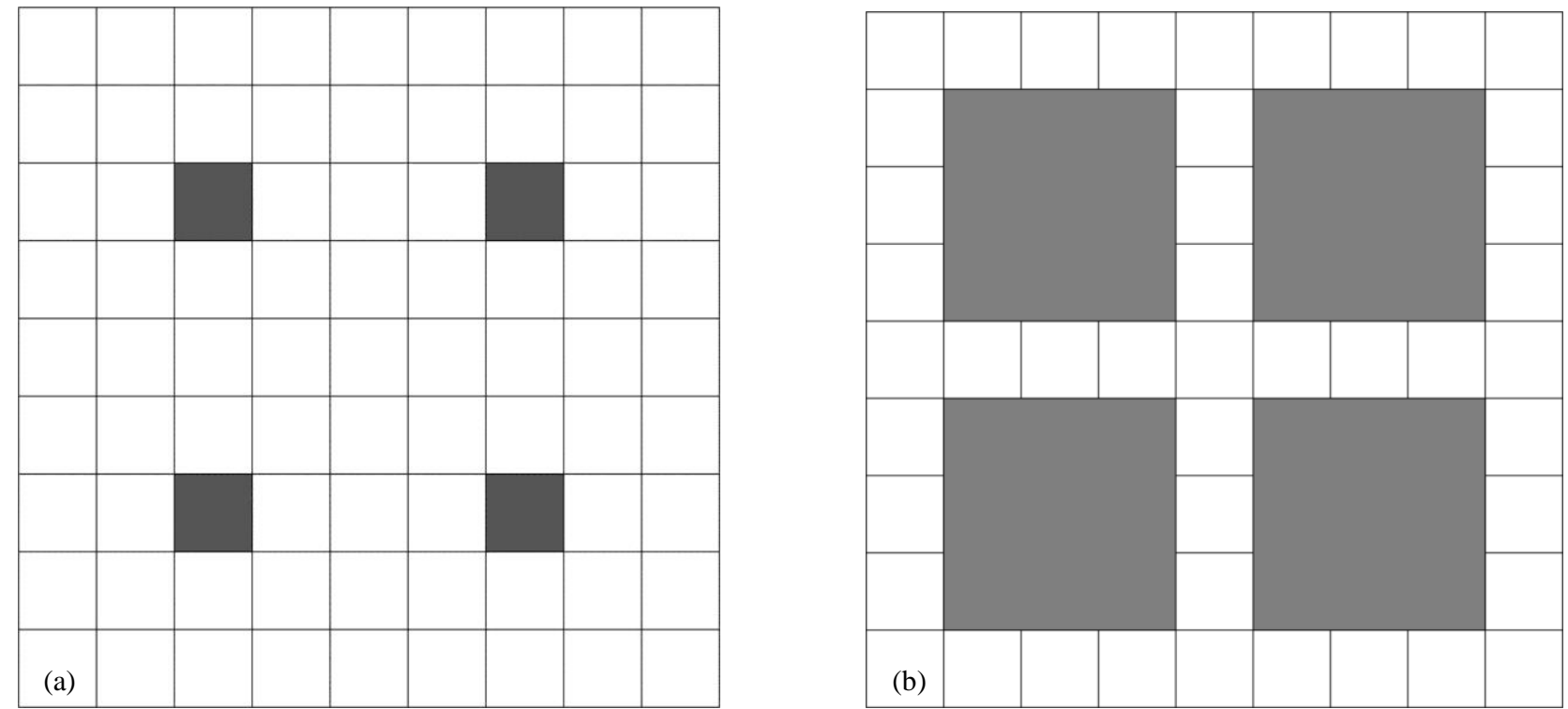

Fig. 3 Schematic representation of the grid points (each small box represents a $25 \mathrm{~km} \times 25 \mathrm{~km} \mathrm{WVC}$ ) used in the global NWP data assimilation: (a) when a thinning of 4 (i.e., one WVC every 4 along and across track WVCs) is applied; (b) when the new low resolution product is used. Note that in the right panel $3 \times 3$ high-resolution WVC winds are averaged to produce the low resolution product.

global NWP, since the representativeness error is spatially correlated. In this section, a lower ASCAT grid resolution is generated for further testing (without thinning) in the ECMWF IFS. Such new product is derived by averaging the winds within an $N \times N$ box (see an example with $N=3$ in Fig. $3 b)$ over the ASCAT $25-\mathrm{km}$ grid product. Since the ASCAT wind product on a $25-\mathrm{km}$ grid is actually of about $50 \mathrm{~km}$ spatial resolution [7], one high-resolution row/column space is left out between neighboring low-resolution grid points in order to avoid instrument-related spatial correlation. In this way, a $3 \times 325-\mathrm{km} \mathrm{WVC}$ wind averaging is actually presented as a $100-\mathrm{km}$ gridded product.

Aggregated observation error SDs are smaller than those from thinned observations due to the lower representativeness error, as shown in Fig. 4. Consequently, the aggregated winds have low representativeness error and thus low error correlation and can be assimilated by the global NWP without thinning.

\section{SUMMARY}

Scatterometer winds are usually thinned and assimilated with low weight to avoid overfitting effects in the global NWP model. However, this leads to a large and quite variable spatial representativeness error which is only statistically accounted for by an enhanced observation error. Several issues are addressed in this study in order to achieve a more effective assimilation of ASCAT winds at ECMWF. First, we increase the amount of triple collocations (buoy-ASCATECMWF) in order to reduce the uncertainties in the error estimation. The ASCAT/ECMWF wind data sets are further separated into 9 categories according to wind quality (or wind variability), and then a thorough analysis is carried out to evaluate the $\mathrm{O} / \mathrm{B}$ errors of the operational ASCAT $25-\mathrm{km}$ and $12.5-\mathrm{km}$ products. Second, several ASCAT lower resolution (or aggregated) products are defined and generated to evaluate their $\mathrm{O} / \mathrm{B}$ errors and to be further tested in the ECMWF data assimilation scheme. The analysis shows that these low resolution products are more representative of the winds at ECMWF scale, and that the observation errors (including the representativeness errors) are also reduced as compared to the operational ASCAT $(25-\mathrm{km}$ and $12.5-\mathrm{km})$ products.

Besides, a suitable wind-speed bias correction is needed because biases are quite detrimental for mesoscale initialization, but exist due to dynamical closure and parameterizations in NWP models. In practice, the scatterometer wind biases are analyzed off-line as a function of wind speed and WVC number, and then a look-up table is created and delivered to the DA system. As such, the probability distribution function of ASCAT wind speed is manipulated simply to be closer to the one of ECMWF wind.

\section{OUTLOOK}

In the near future, impact experiments of the low resolution (aggregated) ASCAT wind products will be carried out at ECMWF. Two months of ASCAT low resolution data (JulyAugust 2015) are used to evaluate the impact of the refined QC and the aggregated or super-obbing technique on the IFS data assimilation system. Besides, several interesting cases, such as that of Fig. 4, will be analyzed in particular to better understand the impact over Tropical regions. To evaluate the impact of each new feature added in this study (i.e., O/B situation-dependent errors, aggregated products), it is proposed to carry out the experiments in an incremental way 

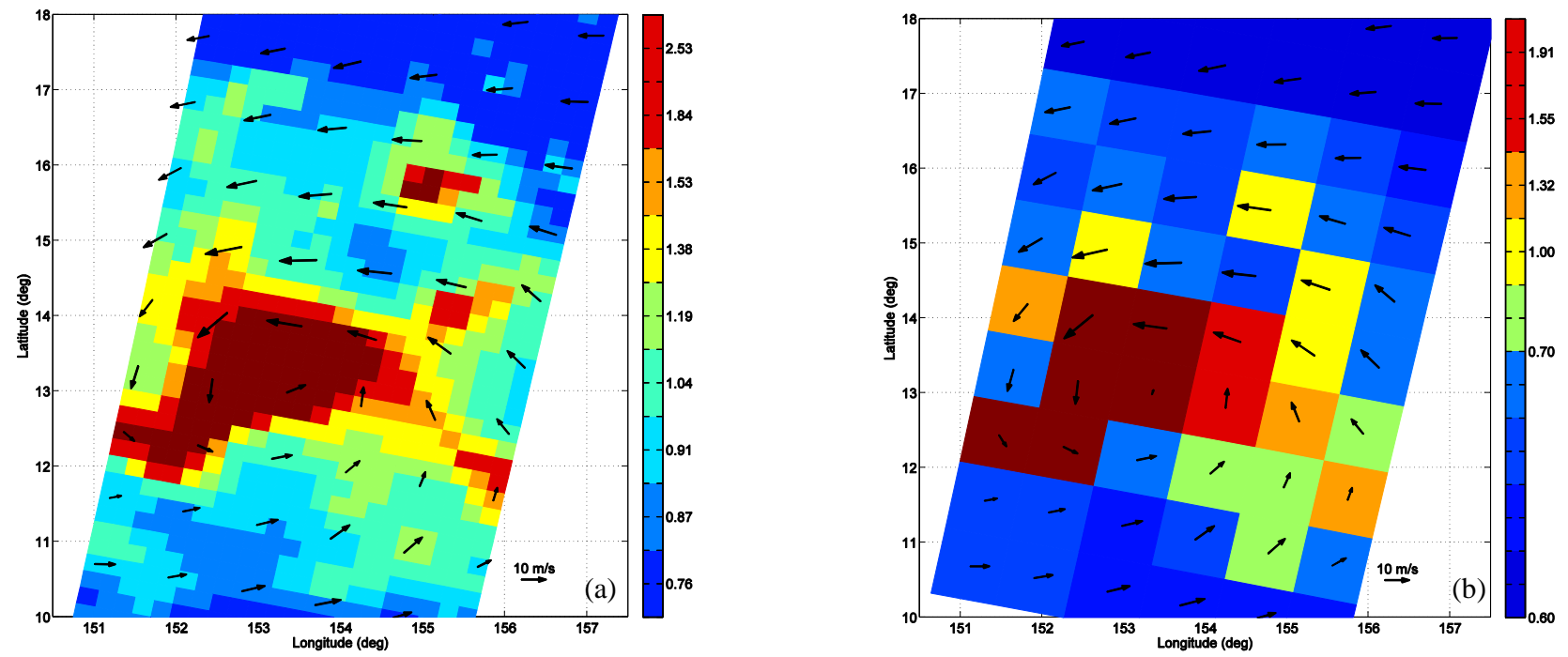

Fig. 4 ASCAT wind fields superimposed on the observation errors (SD): (a) ASCAT 25-km wind field thinned one out of four; (b) ASCAT 100-km low resolution winds derived from the aggregation process.

(i.e., adding one feature at a time). Consequently, the following impact experiments will be carried out:

1) Operational IFS data assimilation approach, using ASCAT-25 km during the mentioned period;

2) Same operational data assimilation, but with the observation errors set according to the strategy proposed in Section 2;

3) New data assimilation with the aggregated $100-\mathrm{km}$ product. In this case, the same amount of grid points and same grid spacing as those of 1) and 2) (except for the two outer WVCs at each swath) are assimilated. Note that a second $180^{\circ}$ ambiguity solution is added for each low resolution grid point, since the current ASCAT assimilation scheme uses two ambiguities $\left(180^{\circ}\right.$ apart) rather than the selected solution.

4) Data assimilation with the $12.5-\mathrm{km}$ coastal product. This high resolution data is thinned by a factor of 16 to $50 \mathrm{~km}$ (i.e., only one WVC out of four WVCs along and across track is used);

5) New data assimilation with a $(3 \times 3) 50-\mathrm{km}$ product and a $(4 \times 4) 62.5-\mathrm{km}$ product aggregated from the $12.5-\mathrm{km}$ coastal product.

\section{REFERENCES}

[1] G. De Chiara, S. English, P. Janssen, and J-R Bidlot, "ASCAT ocean surface wind assessment,"C.D. Jones, and E.F. Roberts, "Article Title," ECMWF Technical Memoranda, Reading, UK, July 2016.
[2] G. De Chiara, L. Isaksen, and S. English, "Assimilation of satellite surface winds at ECMWF," ECMWF/ESA Workshop: Tropical modelling, observations and assimilation, Reading, UK, Nov. 2016.

[3] W. Lin, M. Portabella, A. Stoffelen, J. Vogelzang, and A. Verhoef, "ASCAT wind quality under high sub-cell wind variability conditions," J. Geophys. Res. Oceans, 120, pp.58045819, Aug. 2015.

[4] W. Lin, M. Portabella, A. Stoffelen, J. Vogelzang, and A. Verhoef, "On mesoscale analysis and ASCAT ambiguity removal," Q. J. R. Meteorol. Soc., 142, pp. 1745-1756, Apr. 2016.

[5] A. Stoffelen, J. Figa-Saldaña, A. Cress, J. Cotton, G. De Chiara, T. Valkonen, C. Payan, and G.J. Marseille, "Scatterometer mesoscale NWP data assimilation," 12th International Winds Workshop, IWW12 Splinter discussion session summary, Copenhagen, Denmark, 15-20 June, 2014. http://www.eumetsat.int/website/home/News/ConferencesandEvent s/DAT 2441511.html?lang=EN.

[6] W. Lin, M. Portabella, A. Stoffelen, A. Turiel, and A. Verhoef, "Rain identification in ASCAT winds using singularity analysis," IEEE Trans. Geosci. Remote. Sens. Lett., vol. 11, no. 9, pp. 15191523, Sep. 2014.

[7] EUMETSAT, "Estimation of ASCAT normalised radar cross section: ATBD," EUM/TSS/SPE/14/762689, Jul. 2014. Available: www.eumetsat.int. 